\title{
New Collagen-Dextran-Zinc Oxide Composites for Wound Dressing
}

\author{
Georgeta Păunica-Panea, ${ }^{1,2}$ Anton Ficai, ${ }^{3}$ Minodora Maria Marin, ${ }^{4}$ Ștefania Marin, ${ }^{4}$ \\ Mădălina Georgiana Albu, ${ }^{4}$ Vlad Denis Constantin, ${ }^{1,2}$ Cristina Dinu-Pîrvu, ${ }^{5}$ Zina Vuluga, ${ }^{6}$ \\ Mihai Cosmin Corobea, ${ }^{6}$ and Mihaela Violeta Ghica ${ }^{5}$ \\ ${ }^{1}$ Surgery Department, "Carol Davila” University of Medicine and Pharmacy, Dionisie Lupu 37, 020022 Bucharest, Romania \\ ${ }^{2}$ Surgery Department, "Sfântul Pantelimon" Emergency Clinical Hospital, 340-342 Șoseaua Pantelimon, 021659 Bucharest, Romania \\ ${ }^{3}$ Faculty of Applied Chemistry and Material Science, Politehnica University of Bucharest, 1-7 Polizu Street, 011061 Bucharest, Romania \\ ${ }^{4}$ Collagen Department, Leather and Footwear Research Institute, 93 Ion Minulescu Street, 031215 Bucharest, Romania \\ ${ }^{5}$ Department of Physical and Colloidal Chemistry, Faculty of Pharmacy, "Carol Davila" University of Medicine and Pharmacy 6, \\ Traian Vuia Street, 020956 Bucharest, Romania \\ ${ }^{6}$ Polymer Department, National Institute for Research \& Development in Chemistry and Petrochemistry, \\ 202 Splaiul Independenţei Boulevard, 060021 Bucharest, Romania
}

Correspondence should be addressed to Mihai Cosmin Corobea; mcorobea@yahoo.com

Received 29 September 2015; Accepted 28 December 2015

Academic Editor: Paulo Cesar Morais

Copyright (C) 2016 Georgeta Păunica-Panea et al. This is an open access article distributed under the Creative Commons Attribution License, which permits unrestricted use, distribution, and reproduction in any medium, provided the original work is properly cited.

\begin{abstract}
The goal of this paper was the design, development, and characterization of some new composites, based on collagen and dextran as natural polymers and zinc oxide as antimicrobial, to be used in wound healing. Collagen hydrogels with various concentrations of dextran and zinc oxide were investigated in terms of rheological analysis. The spongious composites, obtained by freeze-drying of hydrogels, were evaluated by morphology (SEM), water uptake, and biological (enzymatic biodegradation) analysis. All the results were strongly influenced by the nature and concentration of composite components. Based on the performances of the hydrogels, stationary rheometry, porous structure, morphology, and biological behavior, the antimicrobial spongious composite based on collagen and dextran with $50 \% \mathrm{ZnO}$ were the most promising for future applications in wound dressing and a biomaterial with high potential in skin regeneration.
\end{abstract}

\section{Introduction}

Wound healing is a significant problem for health-care systems worldwide, accounting over $1.5 \%$ of the world population [1]. The most affected by chronic wounds, as ulcers, are elderly and diabetic people. Moreover, an untreated or not correctly treated wound can lead to large area of necrosis and to systemic infection [2]. To avoid such complications, the best solution is the use of antibacterial biomaterials to treat or prevent infection of the tissues.

Collagen is one of the most used polymers in biomaterials field, due to its excellent properties in biocompatibility, biodegradability, with well-established structure, biologic profile, and in vivo response [3]. It is a bioactive medical device used in different types of injuries (varicose ulcer, burns, wounds, opened surgery, etc.) as haemostatic and medical dressing [4]. Being a natural protein, collagen itself cannot heal the infected tissue because bacteria use it as a substrate [5-7]. Another natural polymer, dextran, a polysaccharide was proven to stimulate wound healing, control the proliferation of bacteria, and affect the metabolism of tumor cells, smooth muscle cells, and endothelial cells [8]. Dextranbased hydrogel containing chitosan microparticles loaded with growth factors [9] and silk fibroin nanofibrous materials with dextran [10] were also successfully used in wound healing, but some simpler solutions were not exploited yet.

In order to induce antimicrobial activity, the polymeric scaffolds have to be more bioactive by decoration with 
antimicrobials like antifungals, antivirals, antiparasitics, or nonpharmaceutical (like essential oils). Zinc oxide ( $\mathrm{ZnO})$ was widely related to exhibit antimicrobial activity and higher stability than organic molecules $[11,12]$. Also, it was used to accelerate the healing of both chronic and acute wounds [13] because of its epithelialization and bacteriostatic properties. $\mathrm{ZnO}$ represents today one of the most reliable choices in obtaining composites with potential applications in wounds care $[14,15]$.

The aim of this study is to develop new simple solutions for wound dressings based on collagen and dextran. The scaffold was designed by using dextran for wound healing and $\mathrm{ZnO}$ for antimicrobial properties. The systems are prepared by lyophilization method in order to obtain efficient absorbent properties for wound dressings and porous structures. The composites in form of hydrogels were evaluated by rheological analysis and the spongious forms were investigated by water uptake, biodegradability in collagenase solution, and SEM.

\section{Materials and Methods}

2.1. Materials and Reagents. Type I fibrillar collagen gel having a concentration of $2.46 \%(\mathrm{w} / \mathrm{w})$ was extracted from calf hide using the technology currently available at the Research-Development Textile Leather National Institute Division Leather and Footwear Research Institute-Collagen Department [3]. Dextran from Leuconostoc spp. (Mw 15,000$25,000)$ was purchased from Fluka (USA) and zinc oxide nanopowder ( $<50 \mathrm{~nm}$ particle size) was from Sigma-Aldrich (USA). Sodium hydroxide and hydrochloric acid were of analytical grade. Type I collagenase obtained from Clostridium histolyticum was purchased from Sigma-Aldrich (Germany) and glutaraldehyde was from Merck (Germany).

2.2. Preparation of Composite Collagen Hydrogels. Dextran and zinc oxide in the concentration given in Table 1 were added to initial collagen gel followed by adjusting collagen concentration at $1 \%$ and $\mathrm{pH} 7.4$ and then cross-linked with $0.25 \%$ glutaraldehyde (related to collagen dry substance).

The composite collagen hydrogels obtained, coded as G1G8, were characterized by rheology and then conditioned by freeze-drying.

2.3. Rheological Analysis and Data Modeling. The stationary shear flow was carried out at $37^{\circ} \mathrm{C}$ using a rotational viscometer MultiVisc-Rheometer Fungilab. The measuring system was equipped with a standard spindle TR 9 and a ThermoHaake P5 Ultrathermostat to maintain the sample temperature at $37 \pm 0.1^{\circ} \mathrm{C}$ during the experiment. The experimental conditions for rheological analysis were previously described [16]. Briefly, before each measurement, the hydrogel was mechanically equilibrated at the aforementioned temperature for about 10 minutes. The flow properties for collagen hydrogels with different concentrations of zinc oxide were assessed applying a rotational speed over the range from 0.3 to $60 \mathrm{rpm}$ that corresponds to a shear rate between 0.1 and $20.4 \mathrm{~s}^{-1}$. The shear stress as a function of shear rate-up curves
TABLE 1: Codification and composition of composite collagen hydrogels.

\begin{tabular}{lccc}
\hline Code of gels & Collagen, $\%$ & Dextran, $\%^{*}$ & Zinc oxide, ${ }^{* *}$ \\
\hline G1 & 1 & 0 & 0 \\
G2 & 1 & 0 & 25 \\
G3 & 1 & 0 & 50 \\
G4 & 1 & 0 & 75 \\
G5 & 1 & 5 & 0 \\
G6 & 1 & 5 & 25 \\
G7 & 1 & 5 & 50 \\
G8 & 1 & 5 & 75 \\
\hline
\end{tabular}

${ }^{*}$ Related to gel volume; ${ }^{* *}$ Related to dry substance of collagen.

was obtained. The rheological data were analyzed applying different models: Bingham (1), Casson (2), Ostwald-de Waele (3), and Herschel-Bulkley (4) $[16,17]$, and the determination coefficients $\left(R^{2}\right)$ values were used as an indicator to select the one that best fitted the upward flow profiles:

$$
\begin{aligned}
\tau & =\tau_{0}+\eta \cdot \dot{\gamma}, \\
\tau^{0.5} & =\tau_{0}{ }^{0.5}+\eta^{0.5} \cdot \dot{\gamma}^{0.5}, \\
\tau & =K \cdot \dot{\gamma}^{n}, \\
\tau & =\tau_{0}+K \cdot \dot{\gamma}^{n},
\end{aligned}
$$

where $\tau$ is shear stress $(\mathrm{Pa}), \dot{\gamma}$ is shear rate $\left(\mathrm{s}^{-1}\right), \eta$ is plastic viscosity $(\mathrm{Pa} \cdot \mathrm{s}), \tau_{0}$ is yield stress $(\mathrm{Pa})$ related to the minimum stress to be applied for determining the start of hydrogel flow, $K$ is consistency index $\left(\mathrm{Pa} \cdot \mathrm{s}^{n}\right)$ associated with the hydrogel viscosity, and $n$ is flow behavior index indicating the flow profiles [18-20].

The Table Curve 2D software was used to evaluate the $R^{2}$ values and rheological parameters specific for each model.

2.4. Preparation of Spongious Collagen Composites. All the composite hydrogels obtained were freeze-dried using a Delta 2-24 LSC lyophilizer (Martin Christ, Germany) and the lyophilization program previously described [21] and porous composites in spongious forms were obtained. The spongious samples were named M1 for the one obtained from G1, M2 for the one obtained from G2, and so on until M8.

2.5. Water Absorption. In order to determine the water absorption, the scaffolds were first immersed in water at $37^{\circ} \mathrm{C}$. At scheduled time intervals, the samples were withdrawn and weighed. The water adsorption was calculated using the following equation:

$$
\text { water uptake }=\frac{W_{t}-W_{d}}{W_{d}} \mathrm{~g} / \mathrm{g},
$$

where $W_{t}$ denotes the weight of the swollen samples at immersion time $t$ and $W_{d}$ denotes the weight of the dry samples (initial samples). All the samples were studied in triplicate. 
TABLE 2: The determination coefficients' values for different rheological models tested at $37^{\circ} \mathrm{C}$.

\begin{tabular}{lccrr}
\hline Rheological models/hydrogels & G1 & G2 & G3 & G4 \\
\hline Casson & 0.9832 & 0.9481 & 0.9418 & 0.9397 \\
Bingham & 0.9233 & 0.8432 & 0.8308 & 0.8168 \\
Ostwald-de Waele & 0.9838 & 0.9904 & 0.9913 & 0.9954 \\
Herschel-Bulkley & 0.9959 & 0.9936 & 0.9931 & 0.9976 \\
\hline
\end{tabular}

TABLE 3: Herschel-Bulkley model fitting parameters for collagen hydrogels with different concentrations of $\mathrm{ZnO}$.

\begin{tabular}{|c|c|c|c|c|}
\hline Rheological parameters/hydrogels & G1 & G2 & G3 & G4 \\
\hline Yield stress $(\mathrm{Pa})$ & 4.707 & 9.704 & 13.559 & 16.406 \\
\hline Consistency index $\left(\mathrm{Pa} \cdot \mathrm{s}^{n}\right)$ & 5.251 & 22.282 & 30.920 & 33.787 \\
\hline Flow index & 0.445 & 0.287 & 0.218 & 0.212 \\
\hline
\end{tabular}

2.6. In Vitro Degradation by Collagenase. In order to investigate the enzymatic degradation of collagen scaffolds, mass loss was monitored as function of exposure time to a collagenase solution. Pieces of $1 \times 1 \mathrm{~cm}$ collagen composites were immersed in a collagenase solution and incubated at $37^{\circ} \mathrm{C}$. At predetermined intervals, the swollen pieces were removed from the collagenase solution and weighed. The percent of degradation was calculated using the following equation:

$$
\% \text { weight loss }=\frac{W_{i}-W_{t}}{W_{t}} \times 100
$$

where $W_{i}$ is the initial weight and $W_{t}$ is the weight of the samples after a time $t$. All the samples were studied in triplicate.

2.7. Scanning Electron Microscopy (SEM). SEM analyses were performed on a HITACHI S2600N electron microscope, on samples covered with silver layer for each collagen composite.

\section{Results and Discussion}

The influence of $\mathrm{ZnO}$ concentration on upward flow curves plotted as shear stress as a function of shear rate for hydrogels $\mathrm{G} 1 \div \mathrm{G} 4$ is presented in Figure 1 .

The upward rheograms presented in Figure 1 indicated that the hydrogels $\mathrm{G} 1 \div \mathrm{G} 4$ showed a non-Newtonian character, the shear stress increase with shear rate increase.

Table 2 summarizes the values obtained for determination coefficients by fitting the experimental data to various rheological models ((1)-(4)) described in Section 2.

As can be seen from Table 1, the Herschel-Bulkley model best fitted the rheological data shear stress as a function of shear rate.

The dependence of $\mathrm{ZnO}$ concentration on the flow descriptors characteristic to Herschel-Bulkley model is given in Table 2.

The data presented in Table 3 show that the hydrogels G1 $\div$ G4 exhibit a pseudoplastic behaviour with yield stress facilitating their flow and allowing their good manipulation $[16,22]$. The values of flow index between 0.212 and 0.445 indicate a high degree of pseudoplasticity, especially for $\mathrm{G} 2-\mathrm{G} 4$. The presence of $\mathrm{ZnO}$ in formulation determined

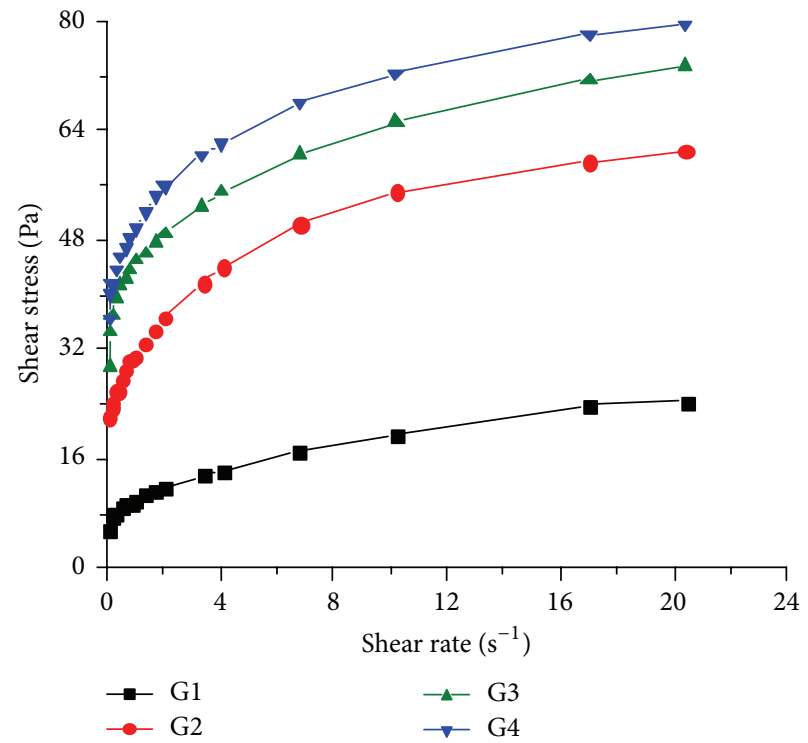

FIGURE 1: The up flow curves for collagen hydrogels with different $\mathrm{ZnO}$ concentrations tested at $37^{\circ} \mathrm{C}$

an increase of consistency index and yield stress, the highest values being recorded for the hydrogel containing a concentration of $75 \% \mathrm{ZnO}$ related to collagen dry substance. The values of the aforesaid descriptors increase with $\mathrm{ZnO}$ concentration, more obvious for the hydrogels with $25 \%$ and $50 \%$ concentration. Thus, the presence of a lower concentration of $\mathrm{ZnO}$ (G2) determined the doubling of yield stress value and also a marked increase of consistency index value (about 4.24 times) compared to sample G1. Moreover, the doubling of $\mathrm{ZnO}$ concentration from $25 \%$ to $50 \%$ determined a more important increase of the rheological parameters for G3 related to G2 (40\% for yield stress and 38\% for consistency index) compared to G4 related to G3, increase from $50 \%$ to $75 \%$, respectively (21\% for yield stress and $38 \%$ for consistency index).

Similar patterns as recorded for $\mathrm{G} 1 \div \mathrm{G} 4$ were obtained for the hydrogels G5 $\div$ G8 with dextran, the flow curves being also described by the Herschel-Bulkley model. The addition of dextran in formulations does not significantly 


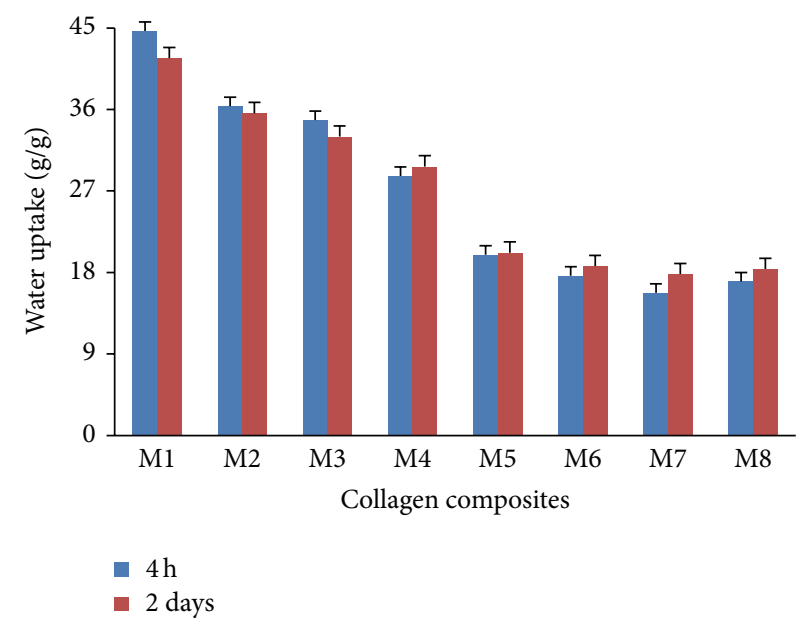

FIGURE 2: Water uptake for collagen composites.

modify rheological parameters specific to the above model for collagen-zinc oxide hydrogels.

The results of the rheological analysis showed that the concentration of zinc oxide is the main factor influencing the flow properties of the designed hydrogels. Thus, beside the own pharmacological effect, zinc oxide concentration significantly affects the flow parameters.

The G1 $\div$ G8 hydrogels were freeze-dried and spongious collagen composites $(\mathrm{M} 1 \div \mathrm{M} 8)$ were obtained and characterized by water absorption, enzymatic degradation, and morphology by SEM.

The water uptake capacity is a very important property for an ideal wound dressing in order to maintain a moist environment and to keep the excessive exudates. Figure 2 presents the water absorption after 4 hours and 2 days for the collagen composites obtained.

The control sample based on cross-linked collagen, M1, had higher water uptake capacity, compared to the ones which contain dextran or zinc oxide. The water uptake decreased as zinc oxide concentration increases from $25 \%$ to $75 \%$ (to collagen content). This behavior could be due to the presence of an increased concentration of zinc oxide which favors a more dense composite structure. The addition of dextran to the samples $M 1 \div \mathrm{M} 4$ decreases the water uptake capacity.

The results were confirmed also by SEM images (Figure 3) which showed the compositional involvement on the spongious composites morphology. According to SEM observations all the scaffolds formed a three-dimensional (3D) porous structure. The pores inside the scaffolds were interconnected and varied in a large range, depending on zinc oxide and dextran content. For M1 (reference sample) the associated morphology (Figure 3(a)) of the structure suggests pore sizes between 80 and $270 \mu \mathrm{m}$, along with the increase of zinc oxide concentration the size of pores decrease to $55-105 \mu \mathrm{m}$ (Figure 3(b)). Moreover $\mathrm{ZnO}$ particles could be clearly seen on collagen fibrils on SEM images. Dextran induced a more homogeneous phase appearance, with smaller pores, with sizes between 48 and $75 \mu \mathrm{m}$ (Figure 3(c)). From Figure 3(d) it can be seen that pores forms were more uniform in the presence of dextran and $\mathrm{ZnO}$ nanoparticles adhered on collagen fibrils (Figures 3(d) and 3(e)).

Both the zinc oxide and the dextran affected the final porous structures of the scaffolds. Materials obtained from collagen hydrogel composites with dextran presented a more "compact" structure and the pore diameter was much smaller compared to the neat collagen ones.

In vitro biodegradation of collagen composites by collagenase solution was assessed to simulate the in vivo behavior of composites used as wound dressings. High degradation rates were registered (Figure 4) for collagen samples without zinc oxide: over $50 \%$ after 4 hours and totally after 2 days.

The dextran content increased the resistance to collagenase and zinc oxide improved the overall stability of the samples. The relative collagen degradation content decreased for samples with $25 \%$ and $50 \% \mathrm{ZnO}$. When large amounts of $\mathrm{ZnO}$ (75\%) were used, the enzymatic degradation slightly increases compared with $50 \% \mathrm{ZnO}$ sample. The same tendency related to composition was observed also for the collagen dextran systems. These results can be explained by the higher amount of minerals which leads to a lower homogenous composite phase; therefore the collagen network can be more exposed to the enzymatic degradation (i.e., on the outer shell of the inorganic particles). Among the composites studied, the most stable ones at both 4 hours and 2 days were the one with collagen, dextran, and 50\% $\mathrm{ZnO}$. Moreover, taking into account the flow analysis for the corresponding hydrogels, the use of $\mathrm{ZnO}$ concentration higher than 50\% was not justified since it does not lead to a marked increase of the rheological parameters and to a higher stability for the porous composites degradation. Considering the wound healing application, the effectiveness of the composite depends on the decreasing collagen rapid degradability profile for prolonged treatment and healing efficiency. 


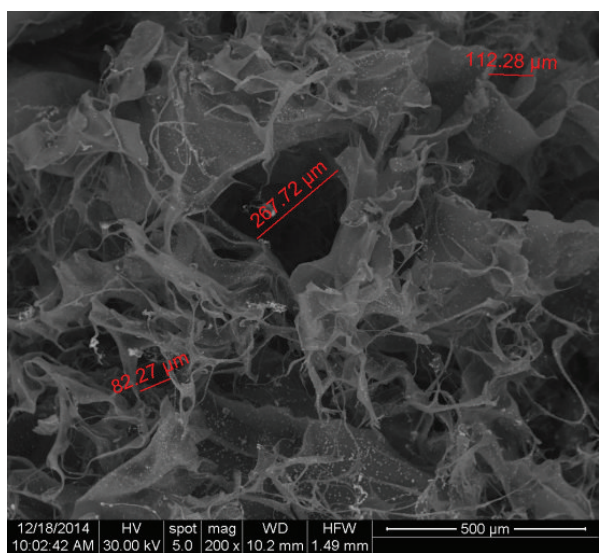

(a)

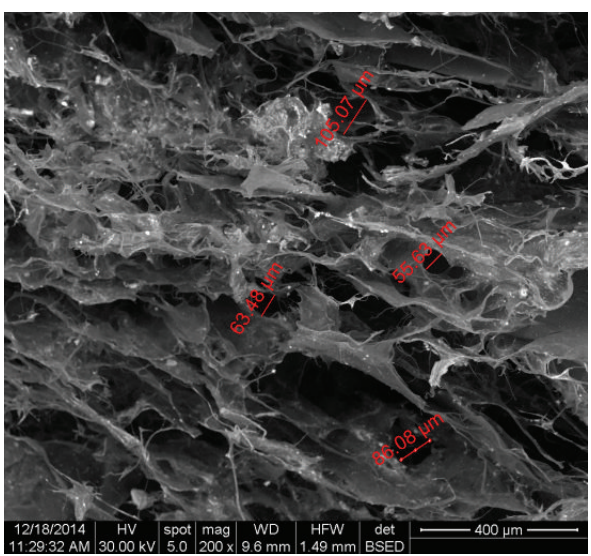

(b)

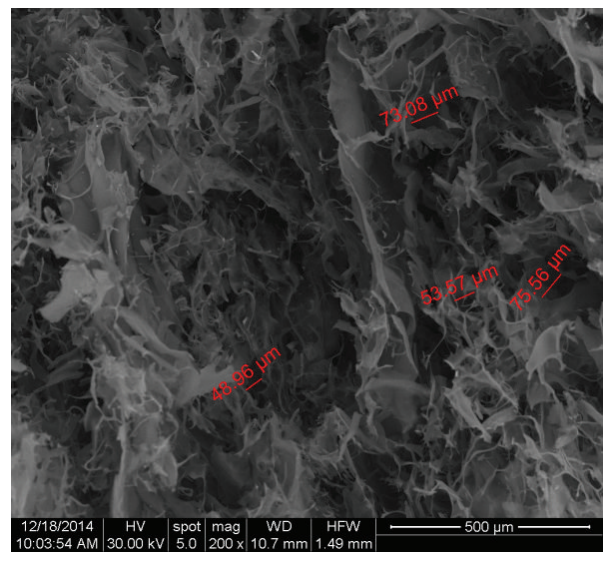

(c)

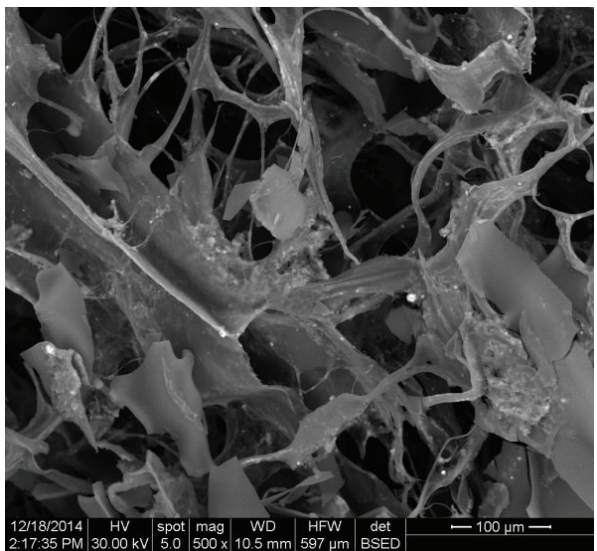

(d)

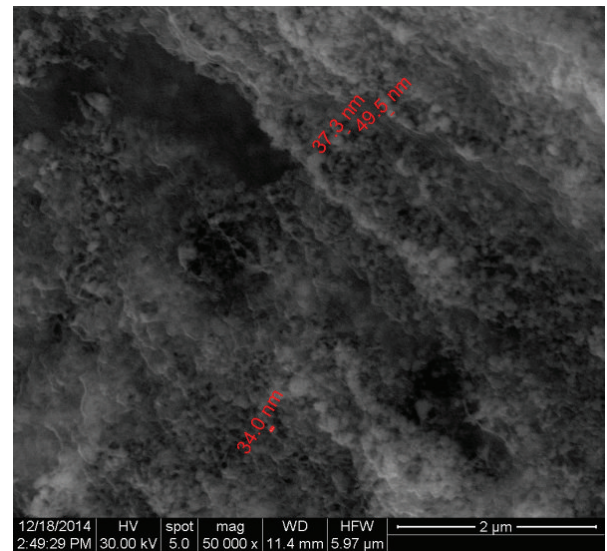

(e)

FIGURE 3: SEM images of collagen composites: (a) M1 (×200); (b) M4 (×200); (c) M5 (×200); (d) M7 (×500); and (e) M7 (×50000).

\section{Conclusions}

$\mathrm{ZnO}$ particles increased the pseudoplastic behavior of the composites based on collagen in solution phase. Dextran presence did not significantly influence the rheological profiles of the hydrogels. $\mathrm{ZnO}$ particles increased the consistency indexes and reduced the flow indexes of solution phases.
The flow parameters indicated the Herschel-Bulkley model for better describing the rheological behavior.

Water uptake ability for the hydrogel composites with dextran and collagen was lower, in comparison with neat collagen ones. $\mathrm{ZnO}$ particles were able to reduce the water uptake for both collagen and collagen/dextran matrixes. 


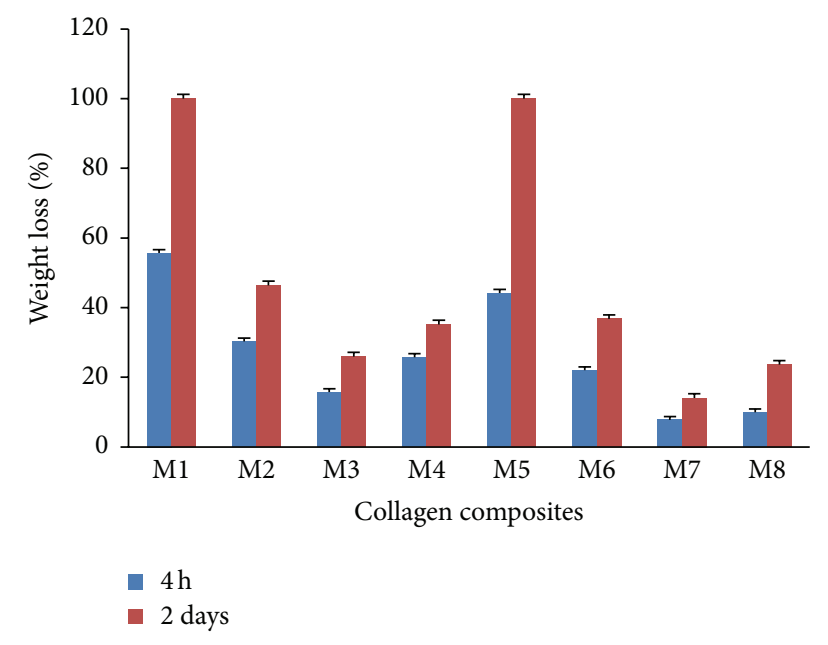

FIGURE 4: In vitro enzymatic degradation of collagen composites.

SEM morphology indicated the decrease of pore sizes and an increase in uniformity when $\mathrm{ZnO}$ was used. The inorganic particles were found in a dispersed state decorating the collagen and collagen/dextran fibrils.

Using $\mathrm{ZnO}$ particles the in vitro degradation profile of the composites can be adjusted by increasing the length of the degradation process (enzymatic assisted). The biodegradability of the composites can be tailored by both dextran and $\mathrm{ZnO}$ particles. But large amounts of $\mathrm{ZnO}$ particles (75\%) can lead to disruption of the composite phase distribution by exposing more collagen to the enzymatic process.

Based on the performances of the hydrogels stationary rheometry and of porous structures morphological and biological investigations, the antimicrobial spongious composite based on collagen and dextran with $50 \% \mathrm{ZnO}$ could be selected for applications to patient wounds, being a promising biomaterial in skin regeneration.

\section{Conflict of Interests}

The authors declare no conflict of interests.

\section{Acknowledgments}

This work was supported by the "Parteneriate in Domenii Prioritare-PNII," supported by MECS-UEFISCDI (Project no. 155/2014) acronym GREENVET. This work received financial support through the project entitled "CEROCareer Profile: Romanian Researcher," Grant no. POSDRU/159/1.5/S/135760, cofinanced by the European Social Fund for Sectoral Operational Programme Human Resources Development 2007-2013.

\section{References}

[1] F. Gottrup, J. Apelqvist, and P. Price, "Outcomes in controlled and comparative studies on non-healing wounds: recommendations to improve the quality of evidence in wound management," Journal of Wound Care, vol. 19, no. 6, pp. 239-268, 2010.
[2] F. De Cicco, E. Reverchon, R. Adami et al., "In situ forming antibacterial dextran blend hydrogel for wound dressing: SAA technology vs. spray drying," Carbohydrate Polymers, vol. 101, no. 1, pp. 1216-1224, 2014.

[3] M. G. Albu, Collagen Gels and Matrices for Biomedical Applications, Lambert Academic Publishing, Saarbrücken, Germany, 2011.

[4] C. A. Fleck and R. Simman, "Modern collagen wound dressings: function and purpose," The Journal of the American College of Certified Wound Specialists, vol. 2, no. 3, pp. 50-54, 2010.

[5] O. Akturk, A. Tezcaner, H. Bilgili, M. S. Deveci, M. R. Gecit, and D. Keskin, "Evaluation of sericin/collagen membranes as prospective wound dressing biomaterial," Journal of Bioscience and Bioengineering, vol. 112, no. 3, pp. 279-288, 2011.

[6] S. Huang and X. Fu, "Naturally derived materials-based cell and drug delivery systems in skin regeneration," Journal of Controlled Release, vol. 142, no. 2, pp. 149-159, 2010.

[7] M. G. Albu, M. Ferdes, D. A. Kaya et al., "Collagen wound dressings with anti-inflammatory activity," Molecular Crystals and Liquid Crystals, vol. 555, pp. 271-279, 2012.

[8] V. Caligur, "Dextran and related polysaccharides," BioFiles, vol. 3, no. 10, article 17, 2008.

[9] M. P. Ribeiro, P. I. Morgado, S. P. Miguel, P. Coutinho, and I. J. Correia, "Dextran-based hydrogel containing chitosan microparticles loaded with growth factors to be used in wound healing," Materials Science and Engineering C, vol. 33, no. 5, pp. 2958-2966, 2013.

[10] M. K. Kim, H. W. Kwak, H. H. Kim et al., "Surface modification of silk fibroin nanofibrous mat with dextran for wound dressing," Fibers and Polymers, vol. 15, no. 6, pp. 1137-1145, 2014.

[11] J. Pasquet, Y. Chevalier, E. Couval, D. Bouvier, and M.-A. Bolzinger, "Zinc oxide as a new antimicrobial preservative of topical products: Interactions with common formulation ingredients," International Journal of Pharmaceutics, vol. 479, no. 1, pp. 88-95, 2015.

[12] L. C. Ann, S. Mahmud, S. K. M. Bakhori et al., "Antibacterial responses of zinc oxide structures against Staphylococcus aureus, Pseudomonas aeruginosa and Streptococcus pyogenes," Ceramics International, vol. 40, no. 2, pp. 2993-3001, 2014.

[13] B. Aksoy, N. Atakan, H. M. Aksoy et al., "Effectiveness of topical zinc oxide application on hypertrophic scar development in rabbits," Burns, vol. 36, no. 7, pp. 1027-1035, 2010.

[14] B. S. Vasile, O. Oprea, G. Voicu et al., "Synthesis and characterization of a novel controlled release zinc oxide/gentamicinchitosan composite with potential applications in wounds care," International Journal of Pharmaceutics, vol. 463, no. 2, pp. 161169, 2014.

[15] P. T. Sudheesh Kumar, V.-K. Lakshmanan, M. Raj et al., "Evaluation of wound healing potential of $\beta$-chitin hydrogel/nano zinc oxide composite bandage," Pharmaceutical Research, vol. 30, no. 2, pp. 523-537, 2013.

[16] M. V. Ghica, M. G. Albu, C. Dinu-Pîrvu, and Şt. Moisescu, "In vitro kinetic release and flow behaviour of some collagenminocycline topical hydrogels," Revista de Chimie, vol. 63, no. 9, pp. 929-935, 2012.

[17] M. G. Albu, M. V. Ghica, L. Popa, M. Leca, and V. Trandafir, "Kinetics of in vitro release of doxycycline hyclate from collagen hydrogels," Revue Roumaine de Chimie, vol. 54, no. 5, pp. 373379, 2009.

[18] M. V. Ghica, Physico-Chemical and Biopharmaceutical Elements of Semisolid Systems with Topical Action. Applications to 
Indomethacin Hydrogels, Printech Publishing House, Bucharest, Romania, 2010.

[19] M. Cespi, G. Bonacucina, S. Pucciarelli et al., "Evaluation of thermosensitive poloxamer 407 gel systems for the sustained release of estradiol in a fish model," European Journal of Pharmaceutics and Biopharmaceutics, vol. 88, no. 3, pp. 954-961, 2014.

[20] J. Ma, Y. Lin, X. Chen, B. Zhao, and J. Zhang, "Flow behavior, thixotropy and dynamical viscoelasticity of sodium alginate aqueous solutions," Food Hydrocolloids, vol. 38, pp. 119-128, 2014.

[21] S. Marin, M. M. Marin, A. M. Ene et al., "Collagen-doxycycline spongious forms for infected tissues treatment," in Proceeding of the 5th International Conference on Advanced Materials and Systems (ICAMS '14), pp. 249-254, Bucharest, Romania, October 2014.

[22] A. Ortan, C. D. Parvu, M. V. Ghica, L. M. Popescu, and L. Ionita, "Rheological study of a liposomal hydrogel based on carbopol," Romanian Biotechnological Letters, vol. 16, no. 1, supplement, pp. 47-54, 2011. 

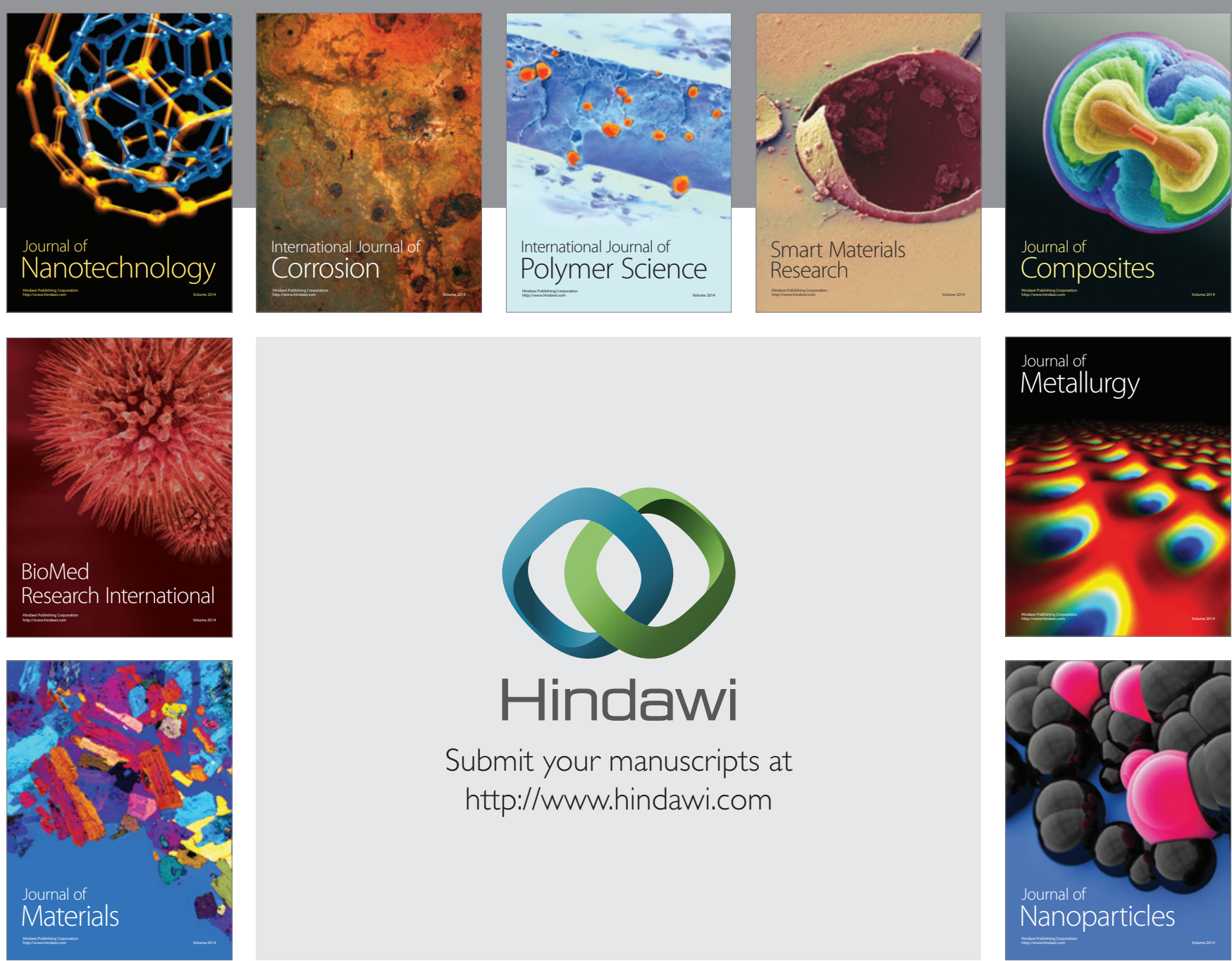

\section{Hindawi}

Submit your manuscripts at

http://www.hindawi.com

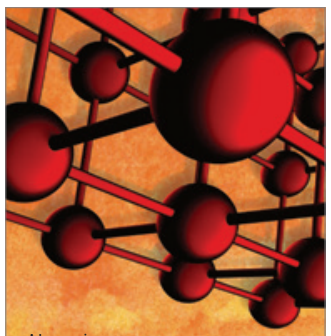

Materials Science and Engineering
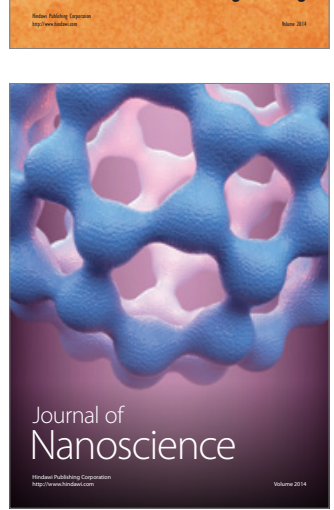
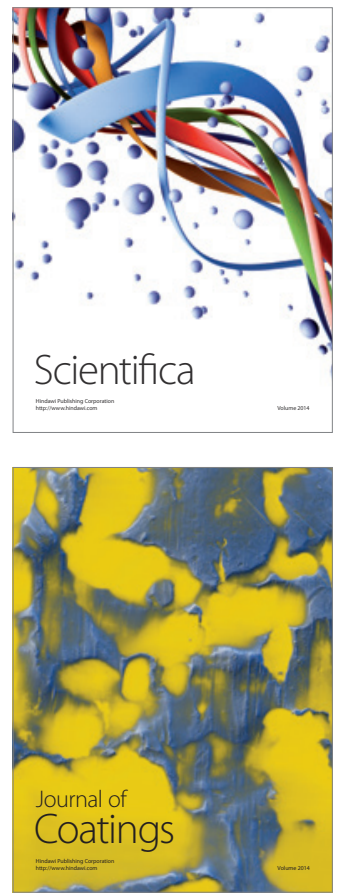
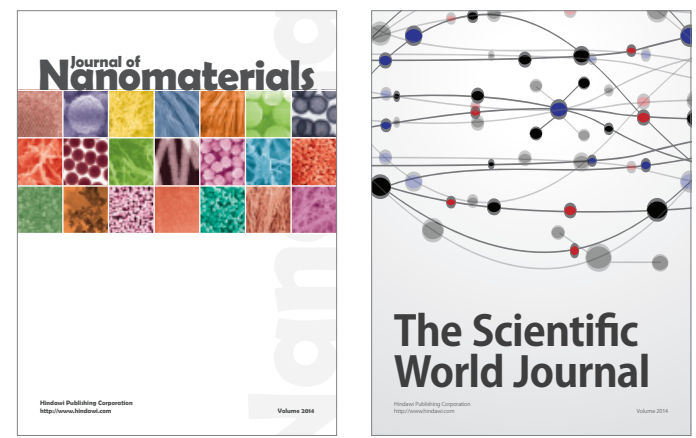

The Scientific World Journal
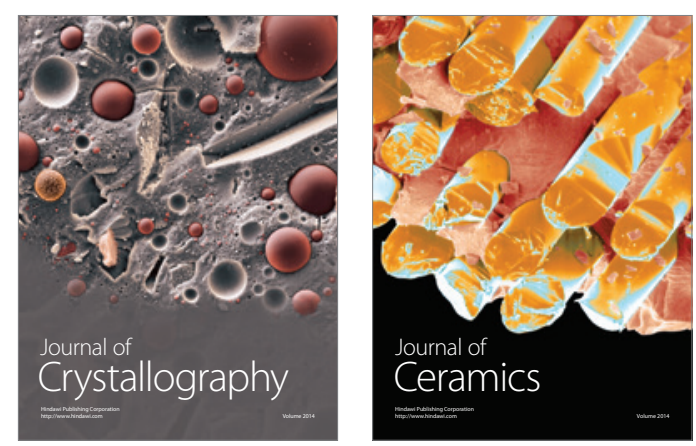
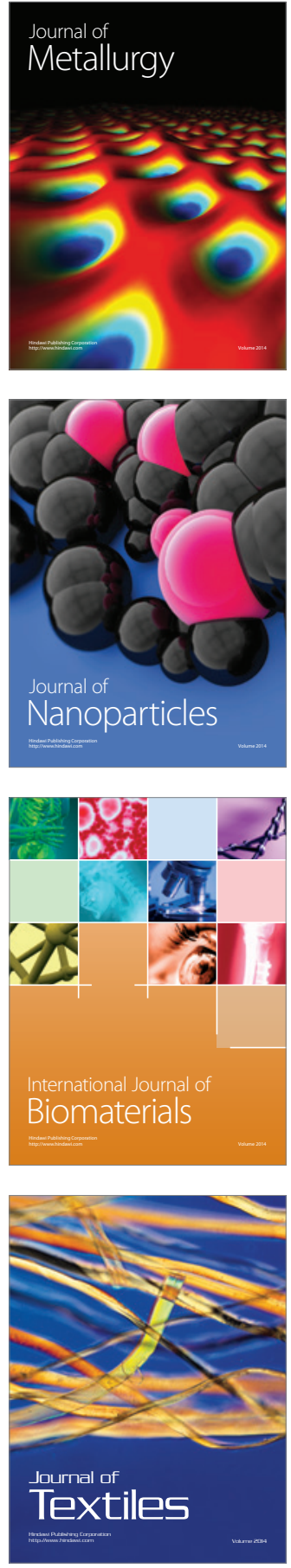